\title{
Hanseníase: pauci e multibacilares estão sendo diferentes?
}

\section{Leprosy: are pauci and multibacillary being diferent?}

\author{
Maria Júlia Izzo Crespo ${ }^{1}$, Aguinaldo Gonçalves ${ }^{2}$, Carlos Roberto Padovani ${ }^{3}$
}

\begin{abstract}
RESUMO
Modelo do estudo: Estudo observacional descritivo transversal. Introdução: A estratégia atual de controle da hanseníase preconizada pela Organização Mundial da Saúde é baseada no respectivo tratamento segundo classificação operacional, se multibacilar ou paucibacilar. Objetivo: Comparar perfis de hansenianos de ambas as formas atendidos em Unidade Hospitalar. Metodologia: Coleta de dados em Fichas de Investigação de Hanseníase do Sistema de Informação de Agravos de Notificação, no Hospital e Maternidade Celso Pierro, PUC Campinas, anos 2007 a 2011. Registro em planilha codificada, armazenamento em banco de dados específico, e processamento estatístico por meio do SPSS. Associações de variáveis investigadas pela prova de Goodman para contraste entre e intra populações multinomiais e pelo teste não-paramétrico de Mann-Whitney, ao nível de $5 \%$ de significância. Resultados: Averiguaram-se 57 casos novos, obtendo-se 14 paucibacilares e 43 multibacilares com indicadores compatíveis com o esperado. No entanto, constataram-se, entre as inconsistências apuradas: i) cinco registros de recidiva, os quais não se confirmaram nosograficamente; ii) caso multibacilar tratado como paucibacilar; iii) quanto a modo de detecção, frequências assemelhadas de encaminhamento e demanda espontânea entre os multibacilares; iv) predomínio de grau zero de incapacidade entre paucibacilares, majoritariamente tuberculóides. Conclusões: Estes resultados indicam a necessidade de vigilância epidemiológica permanente visando à interlocução entre a rede básica e os centros de referência quanto aos procedimentos de controle, com vistas à adequação da gestão técnica dos respectivos Serviços de Saúde.
\end{abstract}

Palavras-Chaves: Hanseníase. Hanseníase/epidemiologia. Perfil Epidemiológico. Classificação. Hanseníase Paucibacilar. hanseníase multibacilar.

\section{Introdução}

Apesar do anúncio da eliminação da hanseníase em nível global pela Organização Mundial da Saúde (OMS) no ano de 2001, a moléstia continua como agravo de saúde pública em muitos países. Em 2011, foram registrados 219.075 (4,06/100 mil habitantes) casos novos da doença no mundo, dentre os quais figuraram 33.955 brasileiros. Em 2012, o Brasil foi o primeiro responsável pela endemia no continente sulamericano e pelo segundo maior número absoluto de casos da doença no mundo. ${ }^{1}$

Trata-se de enfermidade infectocontagiosa crônica associada ao bacilo M.leprae com lesões der-
1. Acadêmica de Medicina, Bolsista do CNPq e da Fundação de Apoio à Pesquisa e Iniciação Científica, PUC-Campinas.

2. Docente Pesquisador, Coordenador, Grupo de Epidemiologia e Saúde, Faculdade de Medicina, Centro de Ciências da Vida, PUC-Campinas.

3. Professor Titular, Instituto de Biociências, UNESP, Campus de Botucatu.
Instituto de Biociências, UNESP, Campus de Botucatu. Distrito de Rubião Júnior, $\mathrm{s} / \mathrm{n}$, CEP 18618-970 - Botucatu, SP - Brasil,

Artigo recebido em 11/07/2013 Aprovado para publicação em 05/12/2013 
matoneurológicas como principais manifestações e alto potencial incapacitante. É considerado caso de hanseníase o portador de uma ou mais das seguintes características: lesão de pele com alteração de sensibilidade; acometimento de nervo com espessamento neural; baciloscopia positiva. Em nossos dias, os princípios básicos para o controle do agravo consistem em diagnóstico precoce e tratamento com poliquimioterapia (PQT). ${ }^{2}$

Em 1982, a OMS instituiu diferentes modalidades de PQT para o tratamento dos casos multibacilares e paucibacilares, $\mathrm{MB}$ e $\mathrm{PB}$, respectivamente, segundo índice bacteriológico (IB) de Ridley. Indivíduos com IB maior ou igual a dois eram considerados $\mathrm{MB}$ e aqueles com IB menor do que dois, PB. Em 1988, já atendendo a necessidades operacionais, os esfregaços positivos tornaram-se suficientes para distinguir MB e, em 1995, a OMS recomendou o critério atualmente em vigência, pelo qual os doentes são alocados de acordo com o número de lesões: PB até cinco lesões, e MB, mais de cinco. Segundo esta segmentação, designada classificação operacional, são utilizadas associações farmacológicas distintas: doentes MBs são tratados durante doze meses com dapsona e clofazimina diariamente e doses mensais supervisionadas de rifampicina e clofazimina; PBs recebem por seis meses dapsona diariamente e rifampicina, administração mensal supervisionada. ${ }^{3}$ Dessa forma, a classificação apropriada é fundamental para tratamento e prognóstico.

São inconveniências do método em vigor de contagem de lesões: necessidade de profissionais treinados para conduzir o exame clínico; inabilidade de investigar todas as partes do corpo por questões culturais; dificuldade, mesmo para experientes, de realizar o diagnóstico em alguns casos, como de lesões hipopigmentadas e sem alteração de sensibilidade, de somente lesões ulceradas ou hiperceratóticas, de distúrbio de sensibilidade localizado sem lesões ou espessamento de nervos, ou em crianças; subjetividade nos achados; ademais, o tamanho das lesões não é considerado. $^{4}$

Para eliminar erros de classificação, bem como para simplificar e abreviar o tratamento da hanseníase, grupo assessor técnico da OMS, em 2002, propôs a uniformização da PQT com esquemas iguais para $\mathrm{PB}$ e $\mathrm{MB}$, consistindo em rifampicina, dapsona e clofazimina por seis meses. ${ }^{5}$ Diante desse panorama, características clínicas e epidemiológicas constituem instrumentos importantes para controle da hanseníase. Isto posto, o presente estudo objetiva comparar perfis de indivíduos MB com $\mathrm{PB}$ atendidos em Unidade Hospitalar e discriminar diferenças identitárias entre ambas as categorias de classificação operacional em campo.

\section{Métodos}

Realizou-se ensaio observacional, quali-quantitativo, descritivo transversal de casos de hanseníase registrados no Hospital e Maternidade Celso Pierro (HMCP), PUC-Campinas, SP, durante todo um quinquênio, de 2007 a 2011, para se poder contar com estudo de base populacional com extensão suficientemente adequada para serem aplicados procedimentos estatísticos validos para grandes amostras. Nesse sentido, o tamanho do grupo considerado foi definido pela totalidade dos pacientes atendidos no período pelo Hospital, não se estendendo a observação para outras instituições exatamente para evitarem-se riscos de perda do controle da qualidade das informações obtidas.

Os dados foram obtidos a partir das Fichas de Notificação/Investigação de Hanseníase (FN/IH), arquivadas no Sistema de Informação de Agravos de Notificação (SINAN), e alimentaram a formulação de planilha de codificação para montagem do respectivo banco, utilizando programa Microsoft Office Excel, versão 2010. Para tanto, adotaram-se como variáveis: Idade, Número de nervos afetados, Intervalo entre diagnóstico e notificação, Intervalo entre diagnóstico e início do tratamento, Número de contatos registrados, Sexo, Número de lesões cutâneas, Forma clínica, Baciloscopia, Modo de entrada, Esquema terapêutico inicial, Modo de detecção e Grau de incapacidade física.

As respostas coletadas foram submetidas à averiguação de coerência interna por: i) seleção daquelas com necessidade de revisão, como elevado número de lesões cutâneas e ocorrência de recidivas; ii) esclarecimento presencial com as funcionárias responsáveis pelo preenchimento do referido instrumento; iii) análise dos respectivos prontuários clínicos; e iv) quando pertinente, convocação e reexame dos doentes, com o deslocamento dos autores para respectivas Unidades de Saúde de origem.

Além disso, também se obteve acesso a partir da Vigilância Sanitária do Distrito de Saúde Noroeste de Campinas à planilha HANSNET, recurso "on line" do SINAN (SINAN Net) construído com a informatização das notificações, que registra todos os casos de hanseníase do município. Para validação do uso das 
FN/IH como ferramenta epidemiológica, compararamse as informações contidas nas mesmas àquelas correspondentes disponíveis na planilha HANSNET.

$\mathrm{O}$ processamento informatizado deu-se pela aplicação do "Statistical Package for Social Sciences". A apresentação estatística dos dados foi realizada de forma descritiva a partir das distribuições de frequência e medidas de posição e variabilidade. ${ }^{6}$ Investigaram-se as associações entre indicadores categorizados pela prova de Goodman para contrastes dentro e entre populações multinomiais ${ }^{7,8}$ e por meio do teste não paramétrico de Mann-Whitney ${ }^{9}$, com apreciações inferenciais ao nível de 5\% de significância. ${ }^{10}$

O projeto de pesquisa origina-se de trabalho mais amplo do Grupo de Pesquisa - "Avaliações Epidemiológicas dos Agravos Dermato-Sanitários: explorando dados primários e secundários" - devidamente aprovado pelo Comitê de Ética em Pesquisa da PUC-Campinas (Protocolo $n^{\circ}$ 0712/11). Dada a ausência de conflito de interesses, contou-se também com a autorização da Secretaria Municipal de Saúde de Campinas.

\section{Resultados}

Como resultado da avaliação de coerência interna, do universo de 57 unidades observacionais identificadas, dez mostraram indicativos de necessidade de averiguação destacada. Critérios e resultados respectivos encontram-se no Quadro 1.
Ademais, durante o processo de coleta e codificação de dados das FN/IH, e a partir da comparação dos mesmos com seus correspondentes informatizados do SINAN Net, observou-se, destacadamente: (1) ausência de preenchimento e uniformidade de resposta nos campos correspondentes às variáveis Ocupação e Acompanhamento respectivamente, com consequente exclusão das mesmas da Planilha de Codificação; (2) uso de versões diferentes da FN/IH com opções de respostas não equivalentes para a mesma variável, o que implicou na conversão das opções de resposta antigas nas suas análogas mais recentes; (3) ocorrências de campos vazios nas FN/IH, mas preenchidos na planilha HANSNET; de campos vazios e erros de cópia/digitação de dados; (4) dificuldade/impossibilidade de recuperação de informações primárias.

A Tabela 1 exibe as medidas descritivas das variáveis quantitativas, indicando que, na comparação entre $\mathrm{PB}$ e $\mathrm{MB}$, não há diferença significativa nas variáveis Idade, Número de nervos afetados, Intervalo entre diagnóstico e notificação, Intervalo entre diagnóstico e início do tratamento e Número de contatos registrados. A casuística é constituída por adultos jovens e há reduzido número de nervos afetados. Quanto ao Intervalo entre diagnóstico e notificação, bem como em relação ao Intervalo entre diagnóstico e início do tratamento, embora os valores das medianas sejam baixos, são apontadas durações extremas elevadas, 109 e 112 dias no segmento

Quadro 1: Critérios e resultados da averiguação de coerência interna na casuística estudada.

\begin{tabular}{c|c|c}
\hline Unidade Observacional & Critério & Resultado \\
\hline 1 & & \\
2 & Registro de recidiva & Recidiva não confirmada \\
3 & & \\
4 & & \\
5 & Registro de 50 lesões & Estimativa numérica compatível com descrição no prontuário. \\
\hline 6 & & \\
\hline 7 & Registro de 20 lesões & Recidiva não confirmada \\
\hline 9 & & \\
\hline
\end{tabular}




\section{Tabela 1}

Medianas (V.mín.; V.máx.) e médias (Desvio Padrão) das variáveis quantitativas estudadas, segundo classificação operacional.

\begin{tabular}{|c|c|c|c|c|c|}
\hline \multirow{3}{*}{$\begin{array}{l}\text { Variável } \\
\text { Idade (anos) }\end{array}$} & \multicolumn{4}{|c|}{ Classificação Operacional } & \multirow{3}{*}{$\begin{array}{c}\text { Valor de } p \\
p>0,05\end{array}$} \\
\hline & \multicolumn{2}{|c|}{ Paucibacilar } & \multicolumn{2}{|c|}{ Multibacilar } & \\
\hline & 49,0 & $(10 ; 61)$ & 47,0 & $(15 ; 81)$ & \\
\hline & 42,6 & $(16,6)$ & 45,9 & $(17,3)$ & \\
\hline \multirow[t]{2}{*}{ Número de nervos afetados } & 0 & $(0 ; 0)$ & 0 & $(0 ; 5)$ & $\mathrm{p}>0,05$ \\
\hline & 0 & (0) & 0,7 & $(1,5)$ & \\
\hline \multirow[t]{2}{*}{ Intervalo entre diagnóstico e notificação (dias) } & 0 & $(0 ; 109)$ & 0 & $(0 ; 324)$ & $\mathrm{p}>0,05$ \\
\hline & 10,3 & $(28,7)$ & 13,6 & $(50,1)$ & \\
\hline \multirow[t]{2}{*}{ Intervalo entre diagnóstico e início do tratamento (dias) } & 00 & $(0 ; 112)$ & $0-0$ & $(0 ; 65)$ & $\mathrm{p}>0,05$ \\
\hline & 9,1 & $(29,7)$ & 4,9 & $(11,9)$ & \\
\hline \multirow[t]{2}{*}{ Número de contatos registrados } & 04,0 & $(1 ; 6)$ & $0-3,0$ & $(0 ; 16)$ & $\mathrm{p}>0,05$ \\
\hline & 3,1 & $(1,6)$ & 3,7 & $(2,9)$ & \\
\hline
\end{tabular}

paucibacilar e 324 e 65 no multibacilar, respectivamente. Ademais, em ambos os grupos, o número de contatos registrados é em torno de três.

A Tabela 2 mostra a distribuição das variáveis qualitativas e discretas investigadas segundo classificação operacional. Nela, dentro de cada coluna de classificação, a presença de pelo menos uma letra diferente, A ou B, na comparação entre as categorias de resposta da variável, indica diferença estatisticamente significante.

\section{Discussão}

Desde pronto, chamam a atenção, entre os resultados adquiridos, cinco registros de recidiva destacados no Quadro 1. Quando submetidos à revisão dirigida, porém, não se confirmaram, quando analisados segundo os critérios correntemente preconizados pelo Ministério da Saúde. Trata-se de situação compatível com os achados de numerosos relatos da literatura, como estudo retrospectivo realizado no Espírito Santo, em que $57,8 \%$ dos casos assim rotulados não poderiam ter este diagnóstico. ${ }^{11}$

Além disso, o presente estudo identificou limitações assinaladas em relação à ferramenta do SINAN utilizada. Paschoal et al. ${ }^{12}$ acrescentam outras ocor- rências: duplicidade de registros, ausência de padronização no lançamento dos dados, falhas de digitação, proveniência de diversas fontes e a partir de múltiplos acessos, perdas, negligência dos funcionários responsáveis pelo preenchimento. Os dados epidemiológicos fornecem substratos para o planejamento das ações de controle e prevenção da doença. Sendo assim, as impropriedades registradas comprometem os resultados e, em consequências, decisões técnicas e, mais amplamente, as respectivas políticas setoriais.

Na Tabela 1, observa-se que MB apresentam maior idade média, nervos afetados mais numerosos, intervalos entre diagnóstico e notificação mais prolongados e número médio de contatos registrados mais elevado, em relação a PB. Estes resultados indicariam maior agravamento da doença em MB, embora, em termos de níveis de significância, as variáveis estudadas não difiram estatisticamente entre os grupos da classificação operacional.

A análise da Tabela 2, por sua vez, permitiu identificar variabilidade de repostas das categorias das variáveis estudadas, segundo classificação operacional, com predomínios bem demarcados, conforme expresso no Quadro 2. Com efeito, nota-se aí que na casuística estudada, MB (43) foram três vezes mais frequentes do que PB (14). Segundo Lana, Carvalho 
Tabela 2

Distribuição das categorias de respostas das variáveis estudadas segundo classificação operacional.

\begin{tabular}{|c|c|c|c|c|}
\hline \multirow[b]{2}{*}{ Variáveis } & \multirow[b]{2}{*}{ Categorias } & \multicolumn{2}{|c|}{ Classificação Operacional } & \multirow[b]{2}{*}{ Valor $p$} \\
\hline & & Paucibacilar & Multibacilar & \\
\hline \multirow[t]{2}{*}{ Sexo } & Feminino & $11(0,786) \mathrm{B}$ & $14(0,326) \mathrm{A}$ & $\mathrm{p}<0,05$ \\
\hline & Masculino & $3(0,214) \mathrm{A}$ & $29(0,674) \mathrm{B}$ & $\mathrm{p}<0,05$ \\
\hline \multirow[t]{4}{*}{ Número de Lesões Cutâneas } & Ausência & $0(0,000) \mathrm{A}$ & $6(0,140) \mathrm{A}$ & $\mathrm{p}<0,05$ \\
\hline & Uma & $10(0,714) \mathrm{BA}$ & $1(0,023) \mathrm{A}$ & $\mathrm{p}<0,05$ \\
\hline & Várias (d"5) & $3(0,214) \mathrm{A}$ & $9(0,209) \mathrm{A}$ & $\mathrm{p}>0,05$ \\
\hline & Muitas (>5) & $1(0,072) \mathrm{A}$ & $27(0,628) \mathrm{B}$ & $\mathrm{p}<0,05$ \\
\hline \multirow[t]{4}{*}{ Forma Clínica } & Indeterminada & $3(0,250) \mathrm{AB}$ & $3(0,070) \mathrm{A}$ & $\mathrm{p}>0,05$ \\
\hline & Tuberculóide & $8(0,667) \mathrm{B}$ & $4(0,093) \mathrm{A}$ & $\mathrm{p}<0,05$ \\
\hline & Dimorfa & $1(0,083) \mathrm{A}$ & $19(0,442) \mathrm{B}$ & $\mathrm{p}<0,05$ \\
\hline & Virchowiana & $0(0,000) \mathrm{A}$ & $17(0,395) \mathrm{B}$ & $\mathrm{p}<0,05$ \\
\hline \multirow[t]{2}{*}{ Bciloscopia } & Positiva & $0(0,000) \mathrm{A}$ & $27(0,658) \mathrm{A}$ & $\mathrm{p}<0,05$ \\
\hline & Negativa & $13(1,000) \mathrm{B}$ & $14(0,342) \mathrm{A}$ & $\mathrm{p}<0,05$ \\
\hline \multirow[t]{3}{*}{ Esquema Terapêutico Inicial } & $\mathrm{PQT} / \mathrm{PB} / 6$ doses & $14(1,000) \mathrm{B}$ & $1(0,023) \mathrm{A}$ & $\mathrm{p}<0,05$ \\
\hline & $\mathrm{PQT} / \mathrm{MB} / 12$ doses & $0(0,000) \mathrm{A}$ & $41(0,954) \mathrm{B}$ & $\mathrm{p}<0,05$ \\
\hline & Outros & $0(0,000) \mathrm{A}$ & $1(0,023) \mathrm{A}$ & $\mathrm{p}>0,05$ \\
\hline \multirow[t]{6}{*}{ Modo de Entrada } & Caso Novo & $13(0,928) \mathrm{B}$ & $33(0,768) \mathrm{B}$ & $\mathrm{p}>0,05$ \\
\hline & Transferência do Mesmo município & $1(0,072) \mathrm{A}$ & $0(0,000) \mathrm{A}$ & $\mathrm{p}>0,05$ \\
\hline & Transferência de Outro município & $0(0,000) \mathrm{A}$ & $3(0,070) \mathrm{A}$ & $\mathrm{p}>0,05$ \\
\hline & Transferência de Outro Estado & $0(0,000) \mathrm{A}$ & $1(0,023) \mathrm{A}$ & $\mathrm{p}>0,05$ \\
\hline & Recidiva & $0(0,000) \mathrm{A}$ & $5(0,116) \mathrm{A}$ & $\mathrm{p}>0,05$ \\
\hline & Outros & $0(0,000) \mathrm{A}$ & $1(0,023) \mathrm{A}$ & $\mathrm{p}>0,05$ \\
\hline \multirow[t]{3}{*}{ Modo de Detecção } & Encaminhamento & $13(0,928) \mathrm{B}$ & $27(0,628) \mathrm{B}$ & $\mathrm{p}<0,05$ \\
\hline & Demanda Espontânea & $1(0,072) \mathrm{A}$ & $14(0,326) \mathrm{B}$ & $\mathrm{p}<0,05$ \\
\hline & Outros & $0(0,000) \mathrm{A}$ & $2(0,046) \mathrm{A}$ & $\mathrm{p}>0,05$ \\
\hline \multirow[t]{3}{*}{ Grau de Incapacidade Física } & Zero & $14(1,000) \mathrm{B}$ & $32(0,744) \mathrm{B}$ & $\mathrm{p}<0,05$ \\
\hline & I & $0(0,000) \mathrm{A}$ & $6(0,140) \mathrm{A}$ & $\mathrm{p}<0,05$ \\
\hline & II & $0(0,000) \mathrm{A}$ & $5(0,116) \mathrm{A}$ & $\mathrm{p}>0,05$ \\
\hline Total & & 14 & 43 & \\
\hline
\end{tabular}


Quadro 2

Predomínios das categorias de respostas das variáveis estudadas, segundo classificação operacional.

\begin{tabular}{lll}
\hline & \multicolumn{2}{c}{ Predomínios } \\
\cline { 2 - 3 } Variáveis & PB & MB \\
\hline Sexo & Mulheres & Homens \\
Número de lesões cutâneas & Uma & Muitas \\
Forma clínica & Tuberculóide & Dimorfa e virchowiana \\
Baciloscopia & Negativa & Positiva e Negativa \\
Esquema terapêutico inicial & PQT-PB & PQT-MB \\
Modo de entrada & Caso novo & Caso novo \\
Modo de detecção & Encaminhamento & Encaminhamento e Demanda espontânea \\
Grau de incapacidade física & Zero & Zero \\
\hline
\end{tabular}

e Davi $(2011)^{13}$, tal resultado poderia significar estabilização da endemia, pelo acometimento de suscetíveis, e diagnóstico tardio, levando a manutenção da cadeia de transmissão.

No que diz respeito ao sexo dos doentes, a maioria dos $\mathrm{MB}$ eram homens, o que pode ser explicado pela maior mobilidade social destes, e, em decorrência, maior risco de exposição ao bacilo, como mencionado por diversos autores. ${ }^{14}$ Coerentemente, o número de mulheres com hanseníase é expressivo, principalmente entre PB (11 de 14), e, em muitos outros estudos, supera o de homens, eventualmente em razão da maior preocupação estética e busca pelos serviços de saúde. ${ }^{15}$

Quanto ao número de lesões cutâneas, mais da metade dos indivíduos possuíam para além de cinco no momento do diagnóstico. Considerando o curso insidioso da doença e, que, na maioria das vezes, o surgimento de lesões cutâneas é responsável pela procura do serviço de saúde, isto sugeriria diagnóstico tardio e/ dificuldade de acesso. ${ }^{16}$ Entretanto, chama atenção o fato de seis de 43 (mais de 13\%) dos MB apresentar ausência de lesões cutâneas, fato também verificado por Souza et al. (2012). ${ }^{17}$ Ademais, houve predomínio de lesão única entre PB e de muitas lesões em MB, o que condiz com a classificação operacional.

A designação PB abriga as formas clínicas indeterminada e tuberculóide e a MB, virchoviana e dimorfa. A indeterminada é encontrada em fase inicial da evolução para os pólos tuberculóide ou virchoviano. A tuberculóide acomete indivíduos resistentes, bem como a dimorfa e a virchoviana implicam perpetuação da transmissão e, no caso, com expressivo poder incapacitante. Os doentes da presente casuística eram predominantemente dimorfos e virchovianos, resultado compatível com os predomínios de multibacilaridade e elevado número de lesões e com outras informações correntes em nosso meio. ${ }^{18}$

$\mathrm{O}$ resultado negativo da baciloscopia não exclui o diagnóstico de hanseníase, que pode ser PB ou MB. Quase metade (27 dos 57) dos exames foi positiva e pertencente a MB. A não inclusão do índice morfológico na rotina dos serviços impede a avaliação da viabilidade dos bacilos e, portanto, do risco de transmissão. ${ }^{19}$

Em relação ao esquema terapêutico inicial, os doentes PB foram prescritos majoritariamente com esquema de seis meses de PQT, e os MB, com a versão alongada de PQT, conforme preconizado pelo MS. ${ }^{20}$ Houve um caso de MB tratado como PB. Com o tratamento dependendo da classificação operacional, $\mathrm{MB}$ podem ser alocados como $\mathrm{PB}$ e, por receber o regime farmacológico destes, favorecer resistência bacteriana e recidivas. De fato, estudo desenvolvido nas Filipinas mostrou que $38-51 \%$ dos pacientes classificados como PB segundo as recomendações da OMS eram MB por meio de critérios histológicos e microbiológicos, estando, portanto, sob risco de subtratamento. $^{21}$

Lembra Scollard (2004) ${ }^{22}$, a simplificação da classificação operacional pode mascarar as verdadeiras relações da resposta imunológica e outros fatores 
genéticos intrínsecos, limitando a informação e inibindo a busca por meios moleculares que apoiem coleta de dados, tratamento e estratégias de controle. De fato, lembram Barreto et al. (2007) ${ }^{23}$, nenhum método, analisado de forma isolada, é sempre $100 \%$ sensível e específico para classificação acurada, quer esse critério seja o número de lesões, a manifestação clínica, o resultado da histopatologia ou da baciloscopia, ou status imunológico/sorológico. Todas essas abordagens não se mostraram suficientes, sendo necessárias novas investidas para que se obtenha classificação mais assertiva, principalmente no que concerne à redução da taxa de recidivas.

No que tange ao modo de entrada, os casos novos corresponderam à maioria dos doentes, em ambas as classificações operacionais, predominância demonstrada há muito por estudos brasileiros. ${ }^{24}$

A respeito do modo de detecção, houve predomínio de encaminhamento entre PB e de encaminhamento e demanda espontânea entre MB. Segundo o Ministério da Saúde ${ }^{2}$, casos novos devem ser diagnosticados, notificados e tratados na Unidade Básica de Saúde e o atendimento hospitalar, restringindo-se a recidivas, necessidade confirmada de cirurgias e indicações de internação. Dessa forma, entre os doentes estudados, deveria predominar somente encaminhamento. A demanda espontânea entre MB indicaria agregação de percepção de maior gravidade às manifestações clínicas objetivas existentes, o que levaria à busca pelo serviço de saúde.

Apesar de a maioria dos doentes (46 de 57) não ter nenhum grau de incapacidade física registrada ao diagnóstico, esta perfaz quase $20 \%$ (11 de 57) e é representada apenas por MB. A ausência de PB dentre os acometidos pode ser traduzida como hipoestimativa, uma vez que a forma tuberculóide, neurítica por excelência, predomina entre estes. A taxa de casos novos com grau II de incapacidade física a cada 100.000 habitantes é indicador recomendado pela OMS na Estratégia Global Aprimorada para Redução Adicional da Carga da Hanseníase (2011-2015), o qual pode ser usado para monitorar o progresso, avaliar a detecção e medir a qualidade dos serviços ${ }^{25}$. Esse grau de incapacidade física está presente em mais de 8,7\% (5 em 57) dos hansenianos da casuística, valor acima dos parâmetros usualmente relatados. ${ }^{26}$ Diante deste resultado e da ausência de incapacidades físicas entre $\mathrm{PB}$, surge o questionamento acerca do significado real do predomínio de grau zero verificado: diagnóstico tardio ou avaliação inadequada dos doentes? ${ }^{27}$
Segundo Ji e Saunderson $(2003)^{28}$, com a adoção do esquema uniforme, doentes com alta positividade bacilar ficariam sob risco de subtratamento e PBs cuja eficácia do atual tratamento não é questionada seriam sobretratados portanto, com adição de custos desnecessários e mais efeitos colaterais. Nesse sentido, ensaio realizado na Índia não encontrou diferenças significativas na melhora clínica dos $\mathrm{PB}$ e, entre os $\mathrm{MB}$, o grupo controle obteve cifras superiores. ${ }^{29}$

Em síntese, com exceção do grau de incapacidade física, o presente estudo mostra que há diferenças nas variáveis consideradas entre PB e MB, apontando que sob esta perspectiva, a uniformização da poliquimioterapia não é interessante.

\section{Conclusão}

Face aos desvios relativos à aplicação da FN/ $\mathrm{IH}$, torna-se imperativo aperfeiçoar seu uso, tendo em vista a impostergável necessidade de informações fidedignas e precisas para subsídio das ações de controle da hanseníase.

$\mathrm{Na}$ presente casuística, confirmam-se as claras diferenças esperadas entre $\mathrm{PB}$ e $\mathrm{MB}$, com exceção das variáveis Modo de detecção e Grau de incapacidade física. Nesta, registrou-se predominância de grau zero concomitante a de forma tuberculóide em PB, apesar da elevada taxa de grau II. Naquela, houve existência de demanda espontânea no predomínio entre MB. Estes resultados, contrários às recomendações da Organização Mundial da Saúde, indicam a necessidade de vigilância epidemiológica permanente visando a interlocução entre a rede básica e os centros de referência quanto aos procedimentos de controle, com vistas à adequação da gestão técnica dos respectivos Serviços de Saúde.

O estudo exploratório revela que existem diferenças identitárias entre $\mathrm{PB}$ e $\mathrm{MB}$, fato que parece não recomendar a uniformização do tratamento.

\section{Agradecimentos}

Os autores expressam sua gratidão aos amigos do Hospital e Maternidade Celso Pierro que viabilizaram a consecução deste Projeto, particularmente o Dr. Ricardo Dutra Sugahara, Diretor Clínico da instituição, pela autorização ao acesso aos dados primários e a Enfa. Samira de Oliveira Santin, do Núcleo de Vigilância Epidemiologica Hospitalar pela generosa acolhida. 


\begin{abstract}
Study design: Cross sectional descriptive observational study. Introduction: Present strategy for leprosy control according to World Health Organization is based on respective treatment for pauci or multibacillary patients. Objectives: To compare leper's profiles cared by an university hospital. Materials and Method: Data collected from Leprosy Notification Protocols, Information System for Notifiable Diseases, Celso Pierro Hospital and Maternity, PUC Campinas, years 2007 to 2011. Registers on codified sheets, storage in specific data base and statistical processing by SPSS program. Associations by Goodman's test for contrasts among multinomial populations and by non-parametric Mann-Whitney's, at 5\% significance level. Results: 57 new cases have been ascertained, 14 paucibacillary and 43 multibacillary, showing clinical epidemiologic behaviors as expected. Nevertheless, inconsistencies have been observed, as: i) five cases of relapse registered, but not clinically confirmed; ii) a multibacillary case treated as paucibacillary; iii) in relation to forms of detection, similar frequencies of recommendation and spontaneous demand in the multibacillary group; iv) predominance of grade zero disabilities in the paucibacillary group. Conclusions: Results point to the necessity of permanent epidemiological surveillance on leprosy control in order to keep enough dialogue between basic units and reference centers.
\end{abstract}

Key-words: Leprosy. Leprosy/epidemiology. Health Profile. Classification. Leprosy, Paucibacillary. Leprosy, Multibacillary.

\section{Referências Bibliográficas}

1.World Health Organization. Global leprosy situation, 2012. Wkly Epidemiol Rec. 2012;87:317-28.

2. BRASIL. Ministério da Saúde. Portaria $n \circ 3.125$, de 7 de outubro de 2010. Aprova as Diretrizes para Vigilância, Atenção e Controle da Hanseníase. Brasília, 2010.

3. Rao PS. Recent advances in the control programs and therapy of leprosy. Indian J Dermatol Venereol Leprol. 2004;70:269-76.

4. Parkash O. Classification of leprosy into multibacillary and paucibacillary groups: an analysis. FEMS Immunol Med Microbiol. 2009;55:1-5.

5. World Health Organization. Report on forth meeting of the WHO technical advisory group on elimination of leprosy. Geneva, 2002.

6. Padovani CR. Estatística na metodologia da investigação científica. Botucatu: Unesp, 1995.

7. Goodman LA. Simultaneous confidence intervals for contrasts among multinomial populations. Ann Math Statist 1964; 35: 716-25.

8. Goodman LA. On simultaneous confidence intervals for multinomial proportions. Technometrics 1965; 7: 247-54.

9. Norman GR, Streiner DL. Biostatistics: the bare essentials. 3th ed. St. Louis: Mosby Year Book, 2008, 393p.

10. Gonçalves A. Os testes de hipótese como instrumental de validação da interpretação (Estatística Inferencial). In: Marconi M.A; Lakatos EM. Técnicas de Pesquisa. São Paulo: Atlas. 1982.

11. Diniz LM, Moreira VM, Puppin MA, Oliveira MLW. Estudo retrospectivo de recidiva da hanseníase no Estado do Espírito Santo. Rev Soc Bras Med Trop. 2009; 42:420-4.

12. Paschoal VDA, Nardi SMT, Cury MRCO, Lombardi C, Virmond MCL, Silva RMDN, et al. Criação de banco de dados para sustentação da pós-eliminação em hanseníase. Ciênc Saúde Coletiva. 2011;16(suppl.1):1201-10.

13. Lana FCF, Carvalho APM, Davi RFL. Perfil epidemiológico da hanseníase na microrregião de Araçuaí e sua relação com as ações de controle. Esc Anna Nery Rev Enferm. 2011;15: 62-7.

14. Lima HN, Sauaia N, Costa VRL, Coelho NGT, Figueiredo PMS. Perfil epidemiológico dos pacientes com hanseníase atendidos em Centro de Saúde em São Luís, MA. Rev Soc Bras Clín Méd. 2010;8:323-7.

15. Miranzi SSC, Pereira LHM, Nunes AP. Perfil epidemiológico da hanseníase em um município brasileiro, no período de 2000 a
2006. Rev Soc Bras Med Trop. 2010;43:62-7.

16. Melão S, Blanco LFO, Mounzer N, Veronezi CCD, Simões PWTA. Perfil epidemiológico dos pacientes com hanseníase no extremo sul de Santa Catarina, no período de 2001 a 2007. Rev Soc Bras Med Trop. 2011;44:79-84.

17. Sousa MWG, Silva DC, Carneiro LR, Almino MLBF, Costa ALF. Perfil epidemiológico da hanseníase no estado do Piauí, período de 2003 a 2008. An Bras Dermatol. 2012;87:389-95.

18. Júnior AFR, Vieira MA, Caldeira AP. Perfil epidemiológico da hanseníase em uma cidade endêmica no Norte de Minas Gerais. Rev Soc Bras Clín Méd. 2012;10:272-7.

19. BRASIL. Ministério da Saúde. Secretaria de Vigilância em Saúde. Departamento de Vigilância Epidemiológica. Guia de procedimentos técnicos: baciloscopia em hanseníase. Brasília: Ministério da Saúde, 2010.

20. BRASIL. Ministério da Saúde. Guia de Vigilância Epidemiológica. $7^{\underline{a}}$ ed. Brasília: Ministério da Saúde, 2010.

21. Pardillo FE, Fajardo TT, Abalos RM, Scollard D, Gelber RH. Methods for the classification of leprosy for treatment purposes. Clin Infect Dis. 2007;44:1096-9.

22. Scollard DM. Classification of leprosy: a full color spectrum, or black and white? Int J Lepr Other Mycobact Dis.2004;72:166-8.

23. Barreto JA, Carvalho CV, Cury Filho M, Garbino JA, Nogueira MES, Soares CT, et al. Hanseníase multibacilar com baciloscopia dos esfregaços negativa: a importância de se avaliar todos os critérios antes. Hansen Int. 2007;32:75-9.

24. Gonçalves A, Gonçalves G, Padovani CR. Estudo de cohorte retrospectiva de hansenianos retratados, com destaque para poliquimioterapia. Hansen Int. 2000;25:31-8.

25. World Health Organization. Enhanced global strategy for further reducing the disease burden due to leprosy: plan period 2011-2015. Geneva, 2009.

26. Gonçalves A. Incapacidades em hanseníase: um estudo da realidade em nosso meio. Hansen Int. 1979; 4:26-35.

27. Virmond M, Duerksen R, Gonçalves A. Report and evaluation of Brazilian experience in the rehabilitation of patients with leprosy. Lepr Rev. 1989;60:214-20.

28. Ji B, Saunderson P. Uniform MDT (U-MDT) regimen for all leprosy patients - another example of wishful thinking. Lepr Rev. 2003;74:2-6.

29. Rao PS, Suneetha S, Pratap DV. Comparative study of uniform-MDT and WHO MDT in Pauci and Multi bacillary leprosy patients over 24 months of observation. Lepr Rev. 2009;80:143-55. 